\title{
Satisfiability-Based Algorithms for Boolean Optimization
}

Vasco M. Manquinho (vmm@sat.inesc.pt)

Dept. of Informatics, Technical University of Lisbon

IST/INESC

Lisbon, Portugal

João P. Marques-Silva (jpms@inesc.pt)

Dept. of Informatics, Technical University of Lisbon IST/INESC/Cadence European Labs.

Lisbon, Portugal

\begin{abstract}
.
This paper proposes new algorithms for the Binate Covering Problem (BCP), a well-known restriction of Boolean Optimization. Binate Covering finds application in many areas of Computer Science and Engineering. In Artificial Intelligence, BCP can be used for computing minimum-size prime implicants of Boolean functions, of interest in Automated Reasoning and Non-Monotonic Reasoning. Moreover, Binate Covering is an essential modeling tool in Electronic Design Automation. The objectives of the paper are to briefly review branch-and-bound algorithms for BCP, to describe how to apply backtrack search pruning techniques from the Boolean Satisfiability (SAT) domain to BCP, and to illustrate how to strengthen those pruning techniques by exploiting the actual formulation of BCP. Experimental results, obtained on representative instances indicate that the proposed techniques provide significant performance gains for a large number of problem instances.
\end{abstract}

Keywords: Binate Covering Problem, Propositional Satisfiability, Branch-and-Bound, Backtrack Search, Non-Chronological Backtracking

\section{Introduction}

The generic Boolean Optimization problem as well as several of its restrictions are well-known computationally hard problems, widely used as modeling tools in Computer Science and Engineering. These problems have been the subject of extensive research work in the past (see for example [1]). In this paper we address the Binate Covering Problem (BCP), one of the restrictions of Boolean Optimization. BCP can be formulated as the problem of finding a satisfying assignment for a given Conjunctive Normal Form (CNF) formula subject to minimizing a given cost function. As with generic Boolean Optimization, BCP also finds many applications, including the computation of minimum-size prime implicants, of interest in Automated Reasoning and Non-Monotonic Reasoning [18], and as a modeling tool in Electronic Design Automation (EDA) $[4,20]$.

(C) 2003 Kluwer Academic Publishers. Printed in the Netherlands.

main.tex; 31/01/2003; $14: 27 ;$ p.1 
In recent years, several powerful search pruning techniques have been proposed for solving BCP, allowing dramatic improvements in the ability to solving large and complex instances of BCP. (Details of the work on BCP can be found in $[4,12,20]$.) Despite these improvements, and as with other NP-hard problems, additional search pruning ability allows in general very significant gains, both in the amount of search and in the run times. The ultimate consequence of proposing new pruning techniques is the potential ability for solving new classes of instances.

The main objective of this paper is to propose additional techniques for pruning the amount of search in branch-and-bound algorithms for solving binate covering problems. These techniques correspond to generalizations and extensions of similar techniques proposed in the Boolean Satisfiability (SAT) domain, where they have been shown to be highly effective $[2,17,22]$. In particular, and to our best knowledge, we provide for the first time conditions which enable branch-and-bound algorithms to backtrack non-chronologically whenever bounding due to the cost function is required to take place. Although our main focus is on one particular bounding mechanism (maximum independent set of clauses), we also establish conditions for non-chronological backtracking with other bounding procedures.

The paper is organized as follows. In Section 2 the notation used throughout the paper is introduced. Afterwards, branch-and-bound covering algorithms are briefly reviewed, giving emphasis to solutions based on SAT algorithms and in section 4 different bounding procedures are also described. In subsequent sections, we propose new techniques for reducing the amount of search. In particular we show how effective search pruning techniques from the SAT domain can be generalized and extended to the BCP domain. Experimental results are presented in Section 8, and the paper concludes in Section 9.

\section{Preliminaries}

An instance $C$ of a covering problem is defined as follows,

$$
\begin{array}{ll}
\text { minimize } & \sum_{j=1}^{n} c_{j} \cdot x_{j} \\
\text { subject to } & A \cdot x \geq b, x \in\{0,1\}^{n}
\end{array}
$$

where $c_{j}$ is a non-negative integer cost associated with variable $x_{j}, 1 \leq$

$j \leq n$ and $A \cdot x \geq b, x \in\{0,1\}^{n}$ denote the set of $m$ linear constraints. If every entry in the $(m \times n)$ matrix $A$ is in the set $\{0,1\}$ and $b_{i}=1,1 \leq$ $i \leq m$, then $C$ is an instance of the unate covering problem (UCP).

main.tex; 31/01/2003; $14: 27 ;$ p.2 
Moreover, if the entries $a_{i j}$ of $A$ belong to $\{-1,0,1\}$ and $b_{i}=1-\mid\left\{a_{i j}\right.$ : $\left.a_{i j}=-1,1 \leq j \leq n\right\} \mid$, then $C$ is an instance of the binate covering problem (BCP). Observe that if $C$ is an instance of the binate covering problem, then each constraint can be interpreted as a propositional clause.

Conjunctive Normal Form (CNF) formulas are introduced next. The use of CNF formulas is justified by noting that the set of constraints of an instance $C$ of $\mathrm{BCP}$ is equivalent to a $\mathrm{CNF}$ formula, and because some of the search pruning techniques described in the remainder of the paper are easier to convey in this alternative representation.

A propositional formula $\varphi$ in Conjunctive Normal Form (CNF) denotes a boolean function $f:\{0,1\}^{n} \rightarrow\{0,1\}$. The formula $\varphi$ consists of a conjunction of propositional clauses, where each clause $\omega$ is a disjunction of literals, and a literal $l$ is either a variable $x_{j}$ or its complement $\bar{x}_{j}$. If a literal assumes value 1 , then the clause is satisfied. If all literals of a clause assume value 0 , the clause is unsatisfied. Clauses with only one unassigned literal are referred to as unit. Finally, clauses with more than one unassigned literal are said to be unresolved. In a search procedure, a conflict is said to be identified when at least one clause is unsatisfied. In addition, observe that a clause $\omega=\left(l_{1}+\cdots+l_{k}\right), k \leq n$, can be interpreted as a linear inequality $l_{1}+\cdots+l_{k} \geq 1$, and the complement of a variable $x_{j}, \bar{x}_{j}$, can be represented by $1-x_{j}$.

When a clause is unit (with only one unassigned literal) an assignment can be implied. For example, consider a propositional formula $\varphi$ which contains clause $\omega=\left(x_{1}+\bar{x}_{2}\right)$ and assume that $x_{2}=1$. For $\varphi$ to be satisfied, $x_{1}$ must be assigned value 1 due to $\omega$. Therefore, we say that $x_{2}=1$ implies $x_{1}=1$ due to $\omega$ or that clause $\omega$ explains the assignment $x_{1}=1$. These logical implications correspond to the application of the unit clause rule [7] and the process of repeatedly applying this rule is called boolean constraint propagation $[17,22]$. It should be noted that throughout the remainder of this paper some familiarity with backtrack search SAT algorithms is assumed. The interested reader is referred to the bibliography (see for example $[1,17]$ for additional references).

Covering problems are often solved by branch-and-bound algorithms $[5,12,20]$. In these cases, each node of the search tree corresponds to a selected unassigned variable and the two branches out of the node represent the assignment of 1 and 0 to that variable. These variables are named decision variables. The first node is called the root (or the top node) of the search tree and corresponds to the first decision level. The decision level of each decision is defined as one plus the decision level of the previous decision.

main.tex; 31/01/2003; $14: 27 ;$ p.3 


\section{Search Algorithms for Covering Problems}

The most widely known approach for solving covering problems is the classical branch-and-bound procedure [20], in which upper bounds on the value of the cost function are identified for each solution to the constraints, and lower bounds on the value of the cost function are estimated considering the current set of variable assignments. The search can be pruned whenever the lower bound estimate is higher than or equal to the most recently computed upper bound. In these

cases we can guarantee that a better solution cannot be found with the current variable assignments and therefore the search can be pruned. The algorithms described in $[5,12,20]$ follow this approach.

Several lower bound estimation procedures can be used, namely the ones based on linear-programming relaxations [12], Lagrangian relaxations [16] or the Log-approximation approach [4]. Nevertheless, and for BCP, the approximation of a maximum independent set of clauses [4] is the most commonly used. The tightness of the lower bounding procedure is crucial for the algorithm's efficiency, because with higher estimates of the lower bound, the search can be pruned earlier. For a better understanding of lower bounding mechanisms, different methods will be described. We will address linear programming relaxations, the Log-approximation approach, and will emphasize the approximation of the maximum independent set of clauses. Covering algorithms also incorporate several powerful reduction techniques, a comprehensive overview of which can be found in $[4,20]$.

With respect to the application of SAT to Boolean Optimization, P. Barth [1] first proposed a SAT-based approach for solving pseudoboolean optimization (i.e. a generalization of $\mathrm{BCP}$ ). This approach consists of performing a linear search on the possible values of the cost function, starting from the highest, at each step requiring the next computed solution to have a cost lower than the most recently computed upper bound. Whenever a new solution is found which satisfies all the constraints, the value of the cost function is recorded as the current lowest computed upper bound. If the resulting instance of SAT is not satisfiable, then the solution to the instance of $\mathrm{BCP}$ is given by the last recorded solution.

Additional SAT-based BCP algorithms have been proposed. In [14] a different algorithmic organization is described, consisting in the integration of several features from SAT algorithms in a branch-and-bound procedure, bsolo, to solve the binate covering problem. The bsolo algorithm incorporates the most significant features from both approaches, namely the bounding procedure and the reduction techniques from

main.tex; $31 / 01 / 2003 ; 14: 27 ;$ p.4 


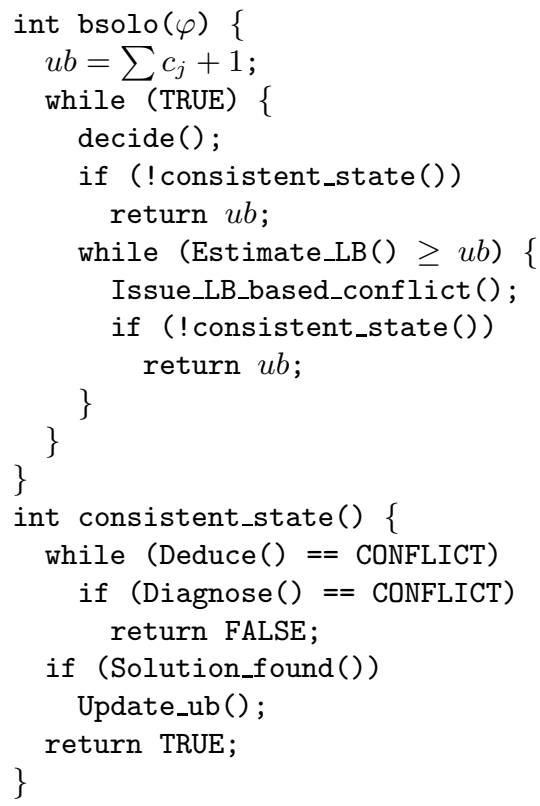

Figure 1. SAT-based branch-and-bound algorithm

branch-and-bound algorithms, and the search pruning techniques from SAT algorithms.

The algorithm presented in [14] already incorporates the main pruning techniques of the GRASP SAT algorithm [17]. Hence, bsolo is a branch-and-bound algorithm for solving BCP that implements a non-chronological backtracking search strategy, clause recording and identification of necessary assignments. Mainly due to an effective conflict analysis procedure which allows non-chronological backtracking steps to be identified, bsolo performs better than other branch-andbound algorithms in several classes of instances, as shown in [14]. However, non-chronological backtracking is limited to one specific type of conflict. In section 5 we describe how to apply non-chronological backtracking to all types of conflicts when using the approximation of a maximum independent set of clauses. Moreover, in section 7 we also describe how to apply the same concepts when using other lower bound estimation methods.

The main steps of a simplified version of the bsolo algorithm (see fig. 1) can be described as follows:

1. Initialize the upper bound to the highest possible value as defined (i.e. given by $u b=\sum_{j=1}^{n} c_{j}+1$ ). 
2. The function consistent_state starts by checking whether the current state yields a conflict. This is done by applying boolean constraint propagation and, in case a conflict is reached, by invoking the conflict analysis procedure, recording relevant clauses and proceeding with the search procedure or backtrack if necessary.

3. If a solution to the constraints has been identified, update the upper bound according to $u b=\sum_{j=1}^{n} c_{j} \cdot x_{j}$. (Observe that the only way to reduce the value of the current solution is to backtrack with the objective of finding a solution with a lower cost.)

4. Estimate a lower bound given the current variable assignments. If this value is higher than or equal to the current upper bound, issue a bound conflict and bound the search by applying the conflict analysis procedure to determine which decision node to backtrack to (using function consistent_state). Continue from step 2.

\subsection{Bound Conflicts}

In bsolo two types of conflicts can be identified: logical conflicts that occur when at least one of the problem instance constraints becomes unsatisfied, and bound conflicts that occur when the lower bound is higher than or equal to the upper bound. When logical conflicts occur, the conflict analysis procedure from GRASP is applied and determines to which decision level the search should backtrack to (possibly in a non-chronological manner).

However, the other type of conflict is handled differently. In bsolo, whenever a bound conflict is identified, a new clause must be added to the problem instance in order for a logical conflict to be issued and, consequently, to bound the search. This requirement is inherited from the GRASP SAT algorithm where, for guaranteeing completeness, both conflicts and implied variable assignments must be explained in terms of the existing variable assignments [17]. With respect to conflicts, each recorded conflict clause is built using the assignments that are deemed responsible for the conflict to occur. If the assignment $x_{j}=1$ (or $x_{j}=$ 0 ) is considered responsible, the literal $\bar{x}_{j}$ (respectively, literal $x_{j}$ ) is added to the conflict clause. This literal basically states that in order to avoid the conflict one possibility is certainly to have instead the assignment $x_{j}=0$ (respectively, $x_{j}=1$ ). Clearly, by construction, after the clause is built its state is unsatisfied. Consequently, the conflict analysis procedure has to be called to determine to which decision level the algorithm must backtrack to. Hence the search is bound.

Whenever a bound conflict is identified, one possible approach to building a clause to bound the search would be to include all decision 
variables in the search tree. In this case, the conflict would always depend on the last decision variable. Therefore, backtracking due to bound conflicts would necessarily be chronological (i.e. to the previous decision level), hence guaranteeing that the algorithm would be complete. Suppose that the set $\left\{x_{1}=1, x_{2}=0, x_{3}=0, x_{4}=1\right\}$ corresponds to all the search tree decision assignments and $\omega_{b c}$ is the clause to be added due to a bound conflict. Then we would have $\omega_{b c}=\left(\bar{x}_{1}+x_{2}+x_{3}+\bar{x}_{4}\right)$. Again, the drawback of this approach (which was used in [14]) is that backtracking due to bound conflicts is always chronological, since it depends on all decision assignments made. In section 5 we propose a new procedure to build these clauses, which enables non-chronological backtracking due to bound conflicts.

\section{Computation of Lower Bounds}

The estimation of lower bounds on the value of the cost function is a very effective method to prune the search tree and the accuracy of lower bounding procedures is critical for identifying areas of the search space where solutions to the constraints with lower values of the cost function cannot be found. In this section we review methods commonly used to estimate a lower bound on the value of the cost function in instances of BCP.

\subsection{Maximum Independent Set of Clauses}

The maximum independent set of clauses (MIS) is a greedy method to estimate a lower bound on the value of the cost function based on an independent set of clauses. (A more detailed definition can be found for example in [4]).

The greedy procedure consists of finding a set $M I S$ of disjoint unate clauses, i.e. clauses with only positive literals and with no literals in common among them. Since maximizing the cost of MIS is an NPhard problem, a greedy computation is used, as shown in fig. 2. The effectiveness of this method largely depends on the clauses included in MIS. Usually, one chooses the clause which maximizes the ratio between its weight and its number of elements.

The minimum cost for satisfying MIS is a lower bound on the solution of the problem instance and is given by,

$$
\begin{aligned}
& \operatorname{Cost}(M I S)=\sum_{\omega \in M I S} W \operatorname{eight}(\omega) \quad \text { where } \\
& W \operatorname{eight}(\omega)=\min _{x_{j} \in \omega} c_{j}
\end{aligned}
$$




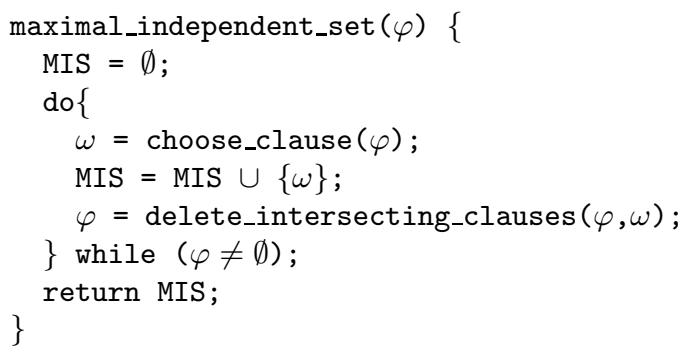

Figure 2. Algorithm for computing a MIS

\subsection{Linear Programming Relaxations}

Linear programming relaxations have long been used as lower bound estimation procedures in branch-and-bound algorithms for solving integer programming problems [16]. For the binate covering problem, the utilization of linear programming relaxations as a lower bound estimation method is proposed in [12]. Moreover, it is also claimed that in most cases the linear programming relaxation (LPR) bound is higher than the one obtained with the MIS approach.

The general formulation of the LPR for a covering problem is obtained from (1) as follows:

$$
\begin{array}{ll}
\text { minimize } & z_{l p r}=\sum_{j=1}^{n} c_{j} \cdot x_{j} \\
\text { subject to } & A \cdot x \geq b \\
& x \geq 0
\end{array}
$$

For simplicity the constraints $x \leq 1$ are not included. The solution of (1) is referred to as $z_{c p}^{*}$, whereas the solution of (4) is referred to as $z_{l p r}^{*}$.

It is well-known that the solution $z_{l p r}^{*}$ of (4) is a lower bound on the solution $z_{c p}^{*}$ of (1) [16]. Basically, any solution of (1) is also a feasible solution of (4), but the converse is not true. Moreover, for a given solution of (4) where $x \in\{0,1\}^{n}$, we necessarily have $z_{c p}^{*}=z_{l p r}^{*}$. Hence, the result follows. Furthermore, different linear programming algorithms can be used for solving (4), some of which with guaranteed worst-case polynomial run time [16].

\subsection{Log-Approximation}

It is well known that the MIS approach can be very far from the minimum cost solution in specific cases of problem instance matrixes [5]. Given that the approximation provided by the greedy algorithm is of 


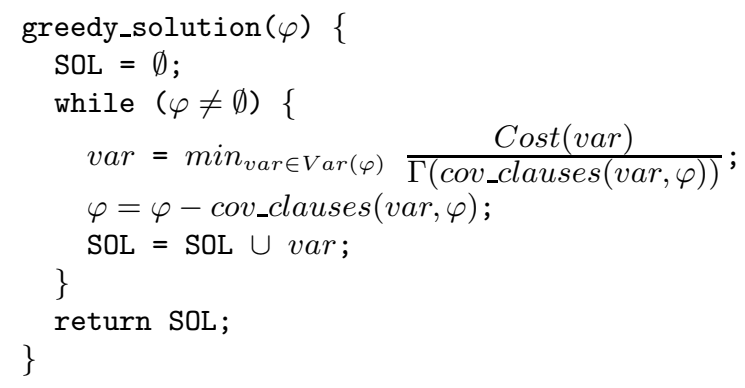

Figure 3. Algorithm for computation of a greedy solution

poor quality, tighter lower bounds can be established. In [5] a new lower bound computation algorithm for unate covering is introduced which guarantees a logarithmic ratio bound on the minimum cost solution.

The algorithm in Fig. 3 describes a procedure for constructing a greedy solution for a covering problem with a set $\varphi$ of constraints to satisfy. At each step a decision variable var is chosen, the clauses that become satisfied by var are removed from $\varphi$ and a solution set is updated. Observe that the algorithm was conceived to tackle unate covering problems [5], but it can be easily modified in order to attempt to find greedy solutions for binate covering instances ${ }^{1}$. The variable to add to the solution set is the one which minimizes the relation between its cost and the value given by $\Gamma$. Let $\gamma$ be a positive weighting function defined on a set of clauses (e.g. the number of free literals). We can define $\Gamma$ as:

$$
\Gamma\left(\varphi^{\prime}\right)=\sum_{\omega \in \varphi^{\prime}} \gamma(\omega)
$$

It can be shown [5] that based on the greedy solution given by the algorithm in Fig. 3, it is possible to obtain a lower bound on the covering problem which is log-approximable to the optimum value $z_{c p}^{*}$. This result is also valid for binate covering whenever the greedy algorithm is able to find a feasible solution. The lower bound is given by:

$$
\begin{gathered}
\frac{\operatorname{Cost}(\mathrm{SOL})}{r} \\
\text { where } r=\sum_{k=1}^{\max _{\varphi^{\prime}} \Gamma\left(\varphi^{\prime}\right)} 1 / k
\end{gathered}
$$

\footnotetext{
${ }^{1}$ In binate covering, the greedy algorithm is unable to guarantee that a solution is found.
}

main.tex; 31/01/2003; $14: 27 ;$ p.9 


\section{SAT-Based Pruning Techniques for BCP}

One of the main features of bsolo is the ability to backtrack nonchronologically when conflicts occur. This feature is enabled by the conflict analysis procedure inherited from the GRASP SAT algorithm. However, as illustrated in section 3.1, in the original bsolo algorithm non-chronological backtracking was only possible for logical conflicts. In the case of a bound conflict all the search tree decision assignments were used to explain the conflict. Therefore, these conflicts would always depend on the last decision level and backtracking would necessarily be chronological.

In this section we describe how to compute sets of assignments that explain bound conflicts. Moreover, we show that these assignments are not in general associated with all decision levels in the search tree; hence non-chronological backtracking can take place.

A bound conflict in an instance of the binate covering problem (BCP) $C$ arises when the lower bound is equal to or higher than the upper bound. This condition can be written as C.path + C.lower $\geq$ C.upper, where C.path is the cost of the assignments already made, C.lower is a lower bound estimate on the cost of satisfying the clauses not yet satisfied (as given for example by an independent set of clauses), and C.upper is the best solution found so far. From the previous equation, we can readily conclude that C.path and C.lower are the unique components involved in each bound conflict. (Notice that C.upper is just the lowest value of the cost function for the solutions of the constraints computed earlier in the search process.) Therefore, we will analyze both C.path and C.lower components in order to establish the assignments responsible for a given bound conflict.

We start by studying C.path. Clearly, the variable assignments that cause the value of C.path to grow are solely those assignments with a value of 1 . Hence, we can define a set of literals $\omega_{c p}$, such that each variable in $\omega_{c p}$ has positive cost and is assigned value 1 :

$$
\omega_{c p}=\left\{l=\bar{x}_{j}: \operatorname{Cost}\left(x_{j}\right)>0 \wedge x_{j}=1\right\}
$$

which basically states that to decrease the value of the cost function (i.e. C.path) at least one variable that is assigned value 1 has instead to be assigned value 0 .

We now consider C.lower. Let MIS be the independent set of clauses, obtained by the method described in section 4.1 , that determines the value of C.lower. Observe that each clause in $M I S$ is part of $M I S$ because it is neither satisfied nor has common literals with any other clause in MIS. Clearly, for each clause $\omega_{i} \in M I S$ these

conditions only hold due to the literals in $\omega_{i}$ that are assigned value

main.tex; 31/01/2003; 14:27; p.10 
0 . If any of these literals was assigned value $1, \omega_{i}$ would certainly not be in MIS since it would be a satisfied clause. Consequently, we can define a set of literals that explain the value of C.lower:

$$
\omega_{c l}=\left\{l: l=0 \wedge l \in \omega_{i} \wedge \omega_{i} \in M I S\right\}
$$

Now, as stated above, a bound conflict is solely due to the two components C.path and C.lower. Hence, this bound conflict will hold as long as the following clause $\omega_{b c}$ is unsatisfied:

$$
\omega_{b c}=\omega_{c p} \cup \omega_{c l}
$$

(Observe that the set union symbol in the previous equation denotes a disjunction of literals.) As long as this clause is unsatisfied, the values of C.path and C.lower will remain unchanged, and so the bound conflict will exist. We can thus use this unsatisfied clause $\omega_{b c}$ to analyze the bound conflict and decide where to backtrack to, using the conflict analysis procedure of GRASP [17]. We should observe that backtracking can be non-chronological, because clause $\omega_{b c}$ does not necessarily depend on all decision assignments. Moreover, due to the clause recording mechanism, $\omega_{b c}$ can be used later in the search process to prune the search tree. If these clauses would depend on all decision assignments, clause recording would not be used since the same set of decision assignments is never repeated in the search process.

Bound conflicts arise during the search process whenever we have C.path + C.lower $\geq$ C.upper. Notice that when a new solution is found, C.lower $=0$ because the independent set is empty (all clauses are satisfied) and C.path is equal to the cost of the new upper bound. Therefore, when we update C.upper with the new value, we have C.path + C.lower $=$ C.upper and a bound conflict is issued in order to backtrack in the search tree. These bound conflicts are just a particular case and the same process we described in this section is applied in order to build the conflict clause.

\section{Reducing Dependencies in Bound Conflicts}

As shown in the previous section, in branch-and-bound BCP algorithms it is possible to establish conditions for implementing non-chronological backtracking due to bound conflicts. However, the ability to backtrack non-chronologically is strongly related with the ability for identifying a small set of assignments that explain each bound conflict. Sets of assignments that include many assignments irrelevant for actually explaining the bound conflict can drastically reduce the ability to backtrack 
non-chronologically. Hence, after computing explanations for bound conflicts, using the techniques described in the previous section, the next step is to identify assignments that can be discarded from each explanation by proving them irrelevant for the bound conflict to take place.

In this section we propose different techniques for reducing dependencies in the explanations of bound conflicts, hence reducing the number of literals in $\omega_{b c}$.

\subsection{Relating C.Path and C.Lower}

Let $l_{j}$ be a literal such that $l_{j} \in \omega_{c p}$ and $l_{j} \notin \omega_{c l}$. Then $l_{j}$ is in $\omega_{b c}$ only due to the C.path component explaining the bound conflict. Let $M I S$ be the independent set, computed with the procedure described in fig. 2, which is used to obtain the value of C.lower. In this situation, literal $l_{j}$ can be removed from $\omega_{c p}$ provided the following conditions apply:

- There exists a satisfied clause $\omega_{i}$ such that $\bar{l}_{j}$ is the only literal which currently satisfies $\omega_{i}$.

- All literals of $\omega_{i}$ besides $l_{j}$ must be positive, unassigned and must not intersect $M I S$ (so that $\omega_{i}$ can be added to $M I S$ if $l_{j}$ assumes value 0$)$.

- All literals in $\omega_{i}$ must have a cost higher than or equal to the cost of literal $l_{j}$.

- No clause in MIS contains $l_{j}$.

This reduction step can be made because if $l_{j}=0, \omega_{i}$ would be in the independent set and the lower bound value would not decrease. Therefore, literal $l_{j}$ can be deemed irrelevant for explaining the bound conflict and can be removed from $\omega_{b c}$.

As an example, let us suppose that variables $x_{1}, x_{2}$ and $x_{3}$ belong to the cost function with the same cost and $x_{1}=1$. If a bound conflict occurs, from (8) $\bar{x}_{1}$ would be in $\omega_{b c}$. However, suppose that clause $\omega_{i}=\left(x_{1}+x_{2}+x_{3}\right)$ is satisfied only due to $x_{1}$, i.e., $x_{2}$ and $x_{3}$ are unassigned. If $x_{2}$ and $x_{3}$ do not belong to any clause in $M I S, \bar{x}_{1}$ can be removed from $\omega_{b c}$ because $x_{1}=1$ is not relevant for the conflict. If variable $x_{1}$ was unassigned or assigned value $0, \omega_{i}$ would be in $M I S$ and the bound conflict would still occur.

It is interesting to observe that we can generalize the second condition, allowing $\omega_{i}$ to have positive literals whose variables are assigned value 0 . Let us consider the example clause $\omega_{i}=\left(x_{1}+x_{2}+x_{3}+x_{4}\right)$. 
Let $x_{1}=1$ and $x_{2}=0$. Moreover, let the cost of $x_{1}$ be no greater than the cost of $x_{2}$, let $x_{3}, x_{4}$ be such that $\omega_{i}$ would be in $M I S$ if $x_{1}=0$, and let no other clause in MIS contain literal $x_{2}$. In this situation, the dependency on $x_{1}$ can be removed, and the dependency on $x_{2}$ need not be considered. Indeed, with $x_{1}=0, \omega_{i}$ would be in $M I S$ and so the cost would not decrease. In addition, since the cost of $x_{2}$ is larger than or equal to the cost of $x_{1}$, by assigning value 1 to $x_{2}$, the cost would also not decrease. Hence the result follows. One should note that the same reasoning applies for an arbitrary number of variables assigned value 0 in a given clause with a single literal assigned value 1.

Next we show how $\omega_{c l}$ can be simplified by evaluating the consequences of modifying the value of some literals on the value of C.path.

Suppose we have a literal $l=x_{j}$, with $l \in \omega_{c l}$ and let $x_{j}=0$. If $x_{j}$ only belongs to one clause $\omega_{i}$ of the independent set and its cost is greater than or equal to the minimum cost of $\omega_{i}$, then $l$ can be removed from $\omega_{b c}$. To better understand how this is possible, suppose instead that $x_{j}=1$. In this situation, $\omega_{i}$ would not be in the independent set (it would be a satisfied clause) and the C.lower component would be lower ${ }^{2}$. However, since the cost of the variable is higher than or equal to the minimum cost of $\omega_{i}$, the C.path component would be higher, and hence the conflict would still hold. So, the assignment $x_{j}=0$ is irrelevant for the conflict to arise and literal $l$ can be removed from $\omega_{b c}$. Observe that the same reasoning still applies even if a clause $\omega_{i}$, containing literal $x_{j}=0$, contains any number of other literals assigned value 0 .

Another reduction technique consists of using a satisfied clause to reduce a dependency from $\omega_{c l}$. Let us consider the following set of clauses,

$$
\begin{aligned}
& \omega_{1}=\left(x_{1}+x_{2}+x_{3}\right) \\
& \omega_{2}=\left(x_{1}+x_{4}+x_{5}\right) \\
& \omega_{3}=\left(\overline{x_{1}}+x_{3}+x_{4}\right)
\end{aligned}
$$

with $x_{1}=0, x_{2}, x_{3}, x_{4}, x_{5}$ unassigned, and let $\omega_{1}$ and $\omega_{2}$ be part of $M I S$. Let the cost of $x_{2}, x_{3}, x_{4}, x_{5}$ be less than or equal to the cost of $x_{1}$. Finally, let no other clause in $M I S$ contain $x_{1}$. If $x_{1}$ would be assigned value 1, C.lower would decrease by 1 since $\omega_{1}$ and $\omega_{2}$ would be satisfied, but $\omega_{3}$ would now be in MIS. However, C.path would be raised due to the cost of $x_{1}$ and the conflict would still hold. Hence, the dependency on $x_{1}$ can be removed.

\footnotetext{
${ }^{2}$ In fact, if the C.lower would be recomputed all over again, it is not guaranteed that it would decrease. Nevertheless, we know that without clause $\omega_{i}$ satisfied by $x_{j}=1, M I S \backslash\left\{\omega_{i}\right\}$ it is still an independent set of clauses. Therefore, $M I S \backslash\left\{\omega_{i}\right\}$ can be used as a low estimate of C.lower.
} 


\subsection{Using Excess Cost Value}

Let us consider a bound conflict and let diff $=($ C.path + C.lower $)-$ C.upper. Clearly, diff $\geq 0$.

It is plain that if C.path was lower by diff, the bound conflict would still hold since we would then have C.path + C.lower $=$ C.upper. Therefore, we may conclude that not all assignments in C.path are necessary for explaining the conflict, since if some assignments were not made, we would still have a bound conflict. In this case, it is possible to remove some literals from $\omega_{c p}$ as long as their cost is lower than or equal to diff.

Moreover, the value of diff can also be used for reducing dependencies from C.lower. Notice that if we remove a subset of clauses D_MIS from MIS (used to obtain C.lower) such that,

$$
\begin{aligned}
& \operatorname{Cost}\left(D_{-} M I S\right) \leq \operatorname{diff} \quad \text { where } \\
& \operatorname{Cost}\left(D_{-} M I S\right)=\sum_{\omega \in D_{-} M I S} W \operatorname{eight}(\omega)
\end{aligned}
$$

then the lower bound conflict will still hold since C.path + C.lower $\geq$ C.upper, where C.lower is now obtained from the independent set of clauses $M I S \backslash D \_M I S$. Therefore, the lower bound conflict clause $\omega_{b c}$ can still be built using (10), but $\omega_{c l}$ can now be reformulated as

$$
\omega_{c l}=\left\{l: l=0 \wedge l \in \omega_{i} \wedge \omega_{i} \in M I S \backslash D \_M I S\right\}
$$

Moreover, the simplifications described above for $\omega_{c l}$ can now be applied to the resulting $\omega_{c l}$.

One should note that the reduction of the number of dependencies relies on which clauses we choose to include in $D \_M I S$. If a clause from $M I S$ is selected with assigned literals belonging to $\omega_{b c}$ because of other clauses in $M I S$ or due to $\omega_{c p}$, then the dependencies are exactly the same. Therefore, it is desirable that $D \_M I S$ be a subset of MIS such that the number of dependencies in $\omega_{b c}$ be minimum. Currently, in bsolo, a greedy procedure is used for selecting the clauses to remove from MIS.

\subsection{Resolution-Induced Dependency Reduction}

In this section we illustrate how the resolution operation [19] can be used for establishing conditions that permit the elimination of dependencies. We should note that the proposed conditions, even though based on the resolution operation, do not require the explicit creation of new clauses.

main.tex; 31/01/2003; 14:27; p.14 
The conditions proposed subsequently can be applied to removing dependencies from $\omega_{c p}$ and $\omega_{c l}$. In all cases, we use examples to illustrate the application of resolution, but provide the necessary conditions for generic application.

We start by studying simplifications to $\omega_{c p}$ established with the resolution operation. Let us consider the following set of clauses,

$$
\begin{aligned}
& \omega_{1}=\left(x_{1}+x_{2}+x_{3}\right) \\
& \omega_{2}=\left(\overline{x_{1}}+x_{2}+x_{4}\right)
\end{aligned}
$$

with $x_{2}=1$, and such that $x_{3}, x_{4}$ are not covered by the currently computed MIS. $x_{1}$ can either be assigned or unassigned, and can either be or not be covered by the currently computed MIS. By applying resolution between $\omega_{1}$ and $\omega_{2}$, with respect to $x_{1}$, we obtain the resulting clause $\omega_{3}=c\left(\omega_{1}, \omega_{2}, x_{1}\right)=\left(x_{2}+x_{3}+x_{4}\right)$. Now, $\omega_{3}$ is certainly satisfied solely by $x_{2}$. Hence, we can conclude that the dependency on $x_{2}$ can be removed by applying the results of sections 6.1 and 6.2 on simplifying $\omega_{c p}$. Notice that $x_{1}$ can be any variable. However, if $x_{1}$ is unassigned and not covered by $M I S$, then we can immediately apply the previous results on simplifying $\omega_{c p}$.

Next, we illustrate one additional form of using the resolution operation for removing dependencies. As an example, assume a bound conflict, and consider the following set of clauses,

$$
\begin{aligned}
& \omega_{1}=\left(x_{1}+x_{2}+x_{3}\right) \\
& \omega_{2}=\left(\overline{x_{1}}+x_{4}+x_{5}\right)
\end{aligned}
$$

where $x_{1}$ is assigned either value 0 or 1 , its cost is 0 , and such that the dependency on $x_{1}$ is only due to $\omega_{1}$ or $\omega_{2}$. Furthermore, let us assume that $\omega_{1}$ would be part of $M I S$ with $x_{1}=0$, and that $\omega_{2}$ would be part of $M I S$ with $x_{1}=1$. In this situation the dependency on $x_{1}$ can be

removed. Notice that if the cost of $x_{1}$ is non-zero, then the removal of the dependency on $x_{1}$ is guaranteed by the previous results (section 6.1) on simplifying $\omega_{c l}$.

Clearly, the application of the resolution operation can be generalized and used for eliminating more than one variable, the only drawback being the computational effort involved.

\section{Bound Pruning with Other Lower Bound Methods}

Sections 5 and 6 describe how to incorporate non-chronological backtracking whenever a bound conflict occurs, either when a new solution is found or when the computed lower bound estimate is higher than or

main.tex; 31/01/2003; 14:27; p.15 
equal to the best solution found so far. Notice that in the first case, the procedure does not depend on the method used for computing the lower bound $^{3}$, whereas in the second case it is assumed that the lower bound computation method is the maximum independent set (MIS) described in section 4.1. If a different lower bound estimation procedure is used, the techniques proposed so far to allow non-chronological backtracking cannot be utilized, since a different procedure will be required for identifying an explanation for the bound conflict. In the remainder of this section we present a theoretical framework which also allows nonchronological backtracking when the other lower bounding procedures described in section 4 are used.

\subsection{Pruning with LP-Relaxation Lower Bounds}

Linear programming relaxations (LPR) are a powerful method to estimate a lower bound value for binate covering instances [12]. However, the resulting backtrack from a bound conflict when using LPR has always been chronological. As mentioned previously, the techniques presented in sections 5 and 6 are useless since the application of the MIS procedure is assumed. The naive approach to build a clause to bound the search in bound conflicts when using LPR would be to include all decision variables in the search tree. However, as stated in section 3.1, the resulting backtrack would necessarily be chronological. In this section, we present a new framework that allows non-chronological backtracking in bound conflicts when linear programming relaxations are used to estimate the lower bound value.

Remember that a bound conflict occurs when C.path + C.lower $\geq$ C.upper, in which case a set of assignments that explains the conflict must be identified. Therefore, we must identify the assignments that explain the value on C.path $\left(\omega_{c p}\right)$ and C.lower $\left(\omega_{c l}\right)$ in order to build the bound conflict clause $\omega_{b c}$ to bound the search. Notice that the value on C.path is independent on the lower bound computation procedure and it can be build as described in section 5 . However, not every reduction technique presented in section 6 can be applied to $\omega_{c p}$, since some of these techniques depend on the fact that the MIS procedure is used to compute C.lower.

The approach to build $\omega_{c l}$ must be different from what was presented in section 5 , since C.lower depends on the value given by the LP-solver. Therefore, the information provided by the LP-solver must be used in order to implement a non-chronological backtracking

\footnotetext{
${ }^{3}$ When a new solution is found, all clauses are satisfied and the lower bound procedure used in the algorithm is irrelevant.
}

main.tex; 31/01/2003; 14:27; p.16 
search strategy in a lower bound conflict when using LPR for bound computation.

Given the value of C.lower using LPR as formulated in section 4.2, let $S$ be the set of constraints with slack ${ }^{4}$ variables assigned value 0 . Observe that these are the constraints which actually limit the value of C.lower, and so will be referred to as the active constraints. When using LP-relaxations to obtain the value of C.lower, the literals that assume value 0 in the active constraints are directly responsible for its value. These literals correspond to the set $\omega_{c l}$ in the bound conflict clause. Applying this reasoning to both assignments of a given variable, allows implementing non-chronological backtracking.

We now illustrate how non-chronological backtrack is possible when using LPR. Let C.lower be computed using LP-relaxations and let $d_{L P R}$ denote the highest decision level, besides the current decision level, of the zero-valued literal assignments in the constraints for which the slack variables assume value 0 . Let the current decision level be $d+k$ and let $d$ be the lowest decision level such that,

$$
\begin{aligned}
& \text { C.path }(d)+C_{l} . \text { lower }(d+k) \geq \text { C.upper } \\
& \text { C.path }(d)+C_{r} . \text { lower }(d+k) \geq C . \text { upper }
\end{aligned}
$$

The highest decision levels involved, besides the current decision level $d+k$ are, respectively, $d_{L P R, l}$ from (17) and $d_{L P R, r}$ from (18). In this situation, the search process can backtrack to decision level $\max \left\{d, d_{L P R, l}, d_{L P R, r}\right\}$. Observe that the highest decision level in $\max \left\{d, d_{L P R, l}, d_{L P R, r}\right\}$ denotes the first possibility for one of the constraints in (17) or (18) not to hold. Hence, the search process can safely and non-chronologically backtrack to this decision level.

\subsection{Pruning with Log-Approximation Lower Bounds}

When C.lower is estimated using the Log-approximation method described in section 4.3, its value depends on the variable assignments chosen by the greedy algorithm (see Fig. 3). Notice that each time a variable assignment is chosen, it depends on the value of function $\Gamma$. Therefore, choosing a variable assignment depends on the clauses which become satisfied with that assignment.

Suppose an assignment to variable $x_{j}$ is chosen at iteration $k$ of the greedy algorithm. This assignment is due to the fact that there is a set of clauses given by cov_clauses $\left(x_{j}, \varphi(k)^{\prime}\right){ }^{5}$ which become satisfied

\footnotetext{
${ }^{4}$ See [3] for a definition of slack and artificial variables.

5 Notice that $\varphi(k)^{\prime}$ denotes the set of clauses still to satisfy at iteration $k$ of the greedy algorithm.
} 
and this set of clauses allows variable $x_{j}$ to be chosen by the algorithm. Therefore, the clauses in cov_clauses provide an explanation for the assignment to variable $x_{j}$. Moreover, the literals assigned value 0 in cov_clauses $\left(x_{j}, \varphi(k)^{\prime}\right)$ are the ones deemed responsible since if they were to have a different value, cov_clauses $\left(x_{j}, \varphi(k)^{\prime}\right)$ would be a smaller set which could cause the assignment to variable $x_{j}$ unrequired and hence C.lower could be lower. Notice that if any of the literals considered responsible were to satisfy some of these clauses by having the opposite value, the set of clauses to satisfy (given by cov_clauses) would be smaller and a lower value for C.lower could be obtained, possibly solving the bound conflict situation.

Let C.lower be estimated using the Log-approximation method and let $S O L$ be the solution found by the greedy algorithm in $n$ iterations which yields a bound conflict. In that case, a bound conflict clause $\omega_{b c}$ must be created to bound the search. The explanation on C.path is determined as described previously in section 5 , since it does not depend on the lower bound estimation method. Moreover, the explanation on C.lower is given by:

$$
\omega_{c l}=\left\{l: l=0 \wedge l \in \omega_{i} \wedge \omega_{i} \in \pi(n)\right\}
$$

where $\pi(n)$ is the set of all clauses covered by the assigned variables chosen until iteration $n$ which is equivalent to:

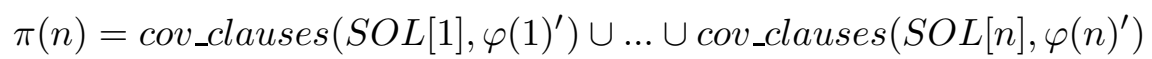

where $S O L[k]$ is the selected assignment at iteration $k$ in the greedy algorithm.

Notice that at iteration $n$ all clauses from $\varphi$ (clauses that are not yet satisfied) are in $\pi(n)$, since all are covered at iteration $n$ of the greedy algorithm. Nevertheless, it is possible that the resulting bound conflict clause $\omega_{b c}$ does not depend on the last decision assignment level and non-chronological backtracking can take place.

\section{Experimental Results}

In this section we compare different algorithms for solving $\mathrm{BCP}$ on example instances taken from digital circuit testing problems [8]. Due to space limitations, only the most representative instances are presented.

For the experimental results given below, the CPU times were obtained on a SUN Sparc Ultra I, running at $170 \mathrm{MHz}$, and with 100 MByte of physical memory. In all cases the maximum CPU time that each algorithm was allowed to spend on any given instance was 1 hour. When 
the algorithm was unable to solve the instance due to time restrictions, the best upper bound found at the time is shown. Otherwise, if no upper bound was computed, the reason of failure is shown, which was either due to the time (time) or memory (mem.) limits imposed. In Tables I and II, besides the time taken and the number of decisions made to solve the instances, it is also shown the number of non-chronological backtracks and the highest jump made in the search tree.

The experimental procedure consisted of running a selected set of problem instances with the bsolo algorithm, as described in Sections 3, 5 and 6 . These results are shown in Tables I and II. Here we can see the differences between several levels of computational effort in identifying dependencies in bound conflicts. Level 0 corresponds to Section 3 where bsolo can only backtrack chronologically in bound conflicts, while level 1 corresponds to the identification of dependencies described in Section 5. The techniques for reducing the number of dependencies presented in Sections 6.1 and 6.2 are only considered into level 2. Level 3 differs from the previous level since it also includes the resolution-based dependency reduction techniques from Section 6.3.

In Table I we can clearly observe significant gains due to the fact that non-chronological backtracking in bound conflicts is implemented in level 1. In several cases we can observe the increase on both the number of non-chronological backtracks and the highest jump in the search tree. For example, instance c3540_F20@1 could not be solved with bsolo's level 0, but was solved in less than one third of the given time limit with the identification of dependencies in bound conflicts.

Table II presents the results for levels 2 and 3. For each of these levels, additional gains are observed, mostly due to more non-chronological backtracks. With the application of techniques for reducing the number of dependencies, smaller set of assignments are declared responsible for the bound conflicts and more non-chronological backtracks are possible.

Finally, in Table III we can observe the results of several other algorithms on the same set of instances. Clearly, lp_solve [13] (a generic Integer Linear Programming solver) is unable to solve almost all instances given the time limit. Notice that only in some cases was it able to find an upper bound to problem instances. scherzo [5], a stateof-the-art BCP solver, which incorporates several powerful pruning techniques in a classical branch-and-bound algorithm, is also unable to solve most of the example instances. The SAT-based linear search algorithm opbdp [1] is able to solve most instances, hence suggesting that these instances are well-suited for SAT-based solvers. Notice however that bsolo is faster than opbdp in most examples, and in some cases the improvement exceeds 1 order magnitude. 
Table I. Results for bsolo levels 0 and 1

\begin{tabular}{|c|c|c|c|c|c|c|c|c|c|}
\hline \multirow{3}{*}{$\frac{\text { Benchmark }}{\text { c1908_F469@0 }}$} & \multirow{3}{*}{$\frac{\min .}{-}$} & \multicolumn{4}{|c|}{ Level 0} & \multicolumn{4}{|c|}{ Level 1} \\
\hline & & \multirow{2}{*}{$\frac{\mathrm{CPU}}{\mathrm{ub} 23}$} & \multicolumn{3}{|c|}{ Dec. NCB Jump } & \multirow{2}{*}{$\frac{\mathrm{CPU}}{\mathrm{ub} 13}$} & \multirow{2}{*}{$\begin{array}{r}\text { Dec. } \\
117079\end{array}$} & \multicolumn{2}{|c|}{ NCB Jump } \\
\hline & & & 72211 & 18 & 6 & & & 721 & 9 \\
\hline c1908_F953@0 & 4 & 438.56 & 2228 & 6 & 2 & 237.54 & 1394 & 61 & 10 \\
\hline c3540_F20@1 & 6 & ub 6 & 10539 & 56 & 7 & 1045.14 & 3359 & 218 & 7 \\
\hline c432_F1gat@1 & & 1414.04 & 15844 & 7 & 3 & 575.16 & 14756 & 608 & 53 \\
\hline c432_F37gat@1 & 9 & ub15 & 143452 & 8 & 3 & ub15 & 218136 & 35785 & 21 \\
\hline c499_Fic2@1 & - & ub41 & 1000029 & 0 & 1 & ub41 & 1003200 & 1586 & 3 \\
\hline c6288_F35gat@1 & 4 & 286.07 & 1255 & 0 & 1 & 107.69 & 756 & 41 & 42 \\
\hline c6288_F69gat@1 & 6 & ub6 & 12379 & 7 & 15 & 1413.17 & 4048 & 110 & 41 \\
\hline 9symml_F1@1 & 9 & 8.30 & 351 & 14 & 5 & 7.41 & 335 & 23 & 5 \\
\hline 9symml_F6@0 & 9 & 6.91 & 301 & 13 & 4 & 6.05 & 272 & 23 & 4 \\
\hline alu4_Fj@0 & 6 & 249.89 & 1566 & 11 & 6 & 185.59 & 1292 & 55 & 4 \\
\hline alu4_Fl@1 & 6 & 159.31 & 1036 & 10 & 3 & 146.01 & 999 & 81 & 4 \\
\hline apex2_Fv14@1 & 10 & 20.48 & 974 & 0 & 1 & 20.15 & 908 & 48 & 4 \\
\hline apex2_Fv17@1 & 12 & 27.85 & 1163 & 5 & 4 & 23.38 & 1082 & 70 & 5 \\
\hline duke2_Fv5@1 & 5 & 36.88 & 592 & 6 & 3 & 26.05 & 515 & 52 & 9 \\
\hline duke2_Fv7@0 & 5 & 16.61 & 356 & 0 & 1 & 13.31 & 335 & 33 & 12 \\
\hline misex3_Fa@0 & 9 & 117.19 & 1526 & 9 & 4 & 56.78 & 898 & 83 & 14 \\
\hline misex3_Fb@1 & 8 & 98.25 & 1128 & 10 & 3 & 83.91 & 1038 & 71 & 8 \\
\hline spla_Fv10@0 & 7 & 42.31 & 809 & 7 & 3 & 34.78 & 766 & 104 & 7 \\
\hline spla_Fv14@0 & 8 & 55.00 & 1064 & 1 & 5 & 38.93 & 914 & 120 & 12 \\
\hline
\end{tabular}

\section{Conclusions}

This paper extends well-known search pruning techniques, from the Boolean Satisfiability domain, to branch-and-bound algorithms for solving the Binate Covering Problem. The paper also describes conditions that allow for non-chronological backtracking in the presence of bound conflicts when using maximum independent sets as a lower bound mechanism. To our best knowledge, this is the first time that branchand-bound algorithms are augmented with the ability for backtracking non-chronologically in the presence of conflicts that result from bound conditions. In addition, we have established conditions for reducing the size of bound conflict explanations, which further elicits non-chronological backtracking. Moreover, we also describe how to enable non-chronological backtracking with other bounding procedures, namely linear programming relaxations and Log-approximation [4].

main.tex; $31 / 01 / 2003 ; 14: 27 ;$ p. 20 
Table II. Results for bsolo levels 2 and 3

\begin{tabular}{|c|c|c|c|c|c|c|c|c|c|}
\hline \multirow{3}{*}{$\frac{\text { Benchmark }}{\text { c1908_F469@0 }}$} & \multirow{3}{*}{$\frac{\min .}{-}$} & \multicolumn{4}{|c|}{ Level 2} & \multicolumn{4}{|c|}{ Level 3} \\
\hline & & \multirow{2}{*}{$\frac{\mathrm{CPU}}{\mathrm{ub} 13}$} & \multirow{2}{*}{$\begin{array}{r}\text { Dec. } \\
111277\end{array}$} & \multicolumn{2}{|c|}{$\overline{\text { NCB Jump }}$} & \multirow{2}{*}{$\begin{array}{l}\mathrm{CPU} \\
\mathrm{ub} 13\end{array}$} & \multirow{2}{*}{$\begin{array}{r}\text { Dec. } \\
11386\end{array}$} & \multicolumn{2}{|c|}{ NCB Jump } \\
\hline & & & & 1049 & 7 & & & 1057 & 7 \\
\hline c1908_F953@0 & 4 & 241.04 & 1416 & 65 & 10 & 240.60 & 1416 & 65 & 10 \\
\hline c3540_F20@1 & & 1009.86 & 3221 & 226 & 7 & 907.40 & 2939 & 213 & 7 \\
\hline c432_F1gat@1 & 8 & 540.20 & 14117 & 647 & 53 & 541.48 & 14117 & 647 & 53 \\
\hline c432_F37gat@1 & 9 & ub14 & 286225 & 48534 & 21 & ub14 & 286490 & 48534 & 21 \\
\hline c499_Fic2@1 & - & ub41 & 1003200 & 1586 & 3 & ub41 & 1003200 & 1586 & 3 \\
\hline c6288_F35gat@1 & 4 & $=108.83$ & 756 & 41 & 42 & 44.42 & 555 & 39 & 42 \\
\hline c6288_F69gat@1 & 6 & 970.29 & 3002 & 100 & 41 & 608.99 & 2198 & 94 & 41 \\
\hline 9symml_F1@1 & 9 & 8.02 & 335 & 23 & 5 & 7.51 & 335 & 23 & 5 \\
\hline 9symml_F6@0 & 9 & 6.52 & 272 & 23 & 4 & 6.12 & 272 & 23 & 4 \\
\hline alu4_Fj@0 & 6 & 157.07 & 1116 & 51 & 4 & 145.73 & 1034 & 46 & 5 \\
\hline alu4_Fl@1 & 6 & 145.02 & 1002 & 84 & 5 & 132.75 & 933 & 73 & 5 \\
\hline apex2_Fv14@1 & 10 & 20.21 & 904 & 55 & 4 & 20.41 & 936 & 60 & 4 \\
\hline apex2_Fv17@1 & 12 & 24.94 & 1089 & 68 & 5 & 23.60 & 1058 & 78 & 5 \\
\hline duke2_Fv5@1 & 5 & 24.89 & 495 & 49 & 9 & 26.60 & 495 & 49 & 9 \\
\hline duke2_Fv7@0 & 5 & 13.01 & 333 & 32 & 12 & 12.93 & 332 & 32 & 12 \\
\hline misex3_Fa@0 & 9 & 55.51 & 879 & 81 & 14 & 55.18 & 879 & 81 & 14 \\
\hline misex3_Fb@1 & 8 & 81.40 & 1006 & 69 & 8 & 80.47 & 1006 & 69 & 8 \\
\hline spla_Fv10@0 & 7 & 35.29 & 765 & 106 & 7 & 33.89 & 764 & 106 & 7 \\
\hline spla_Fv14@0 & 8 & 28.32 & 784 & 114 & 10 & 28.23 & 785 & 113 & 10 \\
\hline
\end{tabular}

Preliminary results obtained on several instances of the Binate Covering Problem indicate that the proposed techniques are indeed effective and can be significant for specific classes of instances, in particular for instances of covering problems with sets of constraints that are hard to satisfy.

Future research work will naturally include seeking further simplification of the clauses created for each type of conflict, and generalizing the bsolo algorithm to other boolean optimization problems.

\section{References}

1. P. Barth. A Davis-Putnam Enumeration Algorithm for Linear Pseudo-Boolean Optimization. Technical Report MPI-I-95-2-003, Max Plank Institute for Computer Science, 1995.

2. R. Bayardo Jr. and R. Schrag. Using CSP look-back techniques to solve realworld SAT instances. In Proceedings of the National Conference on Artificial Intelligence, 1997. 
Table III. Algorithm comparison

\begin{tabular}{lrrrrr}
\hline & & \multicolumn{4}{c}{ Algorithms } \\
\cline { 3 - 6 } Benchmark & min. & lp_solve & scherzo & opbdp & bsolo \\
\hline c1908_F469@0 & - & time & time & ub 24 & ub13 \\
c1908_F953@0 & 4 & time & 3424.81 & ub 26 & 240.60 \\
c3540_F20@1 & 6 & time & mem. & ub 13 & 907.40 \\
c432_F1gat@1 & 8 & ub 15 & time & 1148.27 & 541.48 \\
c432_F37gat@1 & 9 & time & time & 3574.44 & ub14 \\
c499_Fic2@1 & - & time & time & ub 41 & ub41 \\
c5315_F43@0 & 3 & 2.6 & 0.92 & 30.38 & 0.67 \\
c5315_F54@1 & 5 & time & mem. & time & 42.06 \\
c6288_F35gat@1 & 4 & time & mem. & 1330.95 & 44.42 \\
c6288_F69gat@1 & 6 & time & mem. & ub 9 & 608.99 \\
9symml_F1@1 & 9 & ub 9 & 28.64 & 2.01 & 7.51 \\
9symml_F6@0 & 9 & ub 9 & 29.44 & 1.59 & 6.12 \\
alu4_Fj@0 & 6 & time & 879.05 & 413.71 & 145.73 \\
alu4_Fl@1 & 6 & time & 1638.98 & 557.14 & 132.75 \\
apex2_Fv14@1 & 10 & ub 10 & mem. & 624.07 & 20.41 \\
apex2_Fv17@1 & 12 & time & mem. & 532.94 & 23.60 \\
duke2_Fv5@1 & 5 & time & mem. & 82.01 & 26.60 \\
duke2_Fv7@0 & 5 & time & mem. & 18.20 & 12.93 \\
misex3_Fa@0 & 9 & time & mem. & 182.41 & 55.18 \\
misex3_Fb@1 & 8 & time & mem. & 983.55 & 80.47 \\
spla_Fv10@0 & 7 & time & mem. & 202.98 & 33.89 \\
spla_Fv14@0 & 8 & time & mem. & 215.79 & 28.23 \\
\hline & & & & &
\end{tabular}

3. M. S. Bazaraa, J. J. Jarvis and H. D. Sherali Linear Programming and Network Flows. 2nd Ed., John Wiley \& Sons, 1989.

4. O. Coudert. Two-Level Logic Minimization, An Overview. Integration, The VLSI Journal, vol. 17(2):677-691, October 1993.

5. O. Coudert. On Solving Covering Problems. In Proceedings of the ACM/IEEE Design Automation Conference, June 1996.

6. O. Coudert and J. C. Madre. New Ideas for Solving Covering Problems. In Proceedings of the ACM/IEEE Design Automation Conference, June 1995.

7. M. Davis and H. Putnam. A Computing Procedure for Quantification Theory. Journal of the Association for Computing Machinery, vol. 7:201-215, 1960.

8. P. F. Flores, H. C. Neto, and J. P. Marques Silva. An exact solution to the minimum-size test pattern problem. In Proceedings of the IEEE International Conference on Computer Design, pages 510-515, October 1998.

9. J. Gimpel. A Reduction Technique for Prime Implicant Tables. IEEE Transactions on Electronic Computers, vol. EC-14:535-541, August 1965.

10. E. Goldberg, L. Carloni, T. Villa, R. K. Brayton, and A. L. SangiovanniVincentelli. Negative thinking by incremental problem solving: application 
to unate covering. In Proceedings of the ACM/IEEE International Conference on Computer-Aided Design, 1997.

11. G. Hachtel and F. Somenzi. Logic Synthesis and Verification Algorithms. Kluwer Academic Pub., 1996.

12. S. Liao and S. Devadas. Solving Covering Problems Using LPR-Based Lower Bounds. In Proceedings of the ACM/IEEE Design Automation Conference, 1997.

13. M. R. C. M. Berkelaar UNIX Manual Page of lp-solve. In Eindhoven University of Technology, Design Automation Section, ftp://ftp.es.ele.tue.nl/pub/lp_solve, 1992.

14. V. M. Manquinho, P. F. Flores, J. P. Marques Silva, and A. L. Oliveira. Prime implicant computation using satisfiability algorithms. In Proceedings of the IEEE International Conference on Tools with Artificial Intelligence, pages 232239, November 1997.

15. D. De Micheli. Synthesis and Optimization of Digital Circuits. McGraw-Hill, 1994.

16. G. L. Nemhauser and L. Wolsey. Integer and Combinatorial Optimization. John Wiley \& Sons, 1988.

17. J. P. Marques Silva and K.Ã. Sakallah. GRASP: A new search algorithm for satisfiability. In Proceedings of the ACM/IEEE International Conference on Computer-Aided Design, pages 220-227, November 1996.

18. C. Pizzuti Computing Prime Implicants by Integer Programming. In Proceedings of the IEEE International Conference on Tools with Artificial Intelligence, pages 332-336, November 1996.

19. S. J. Russell and P. Norvig Artificial Intelligence: A Modern Approach. Prentice-Hall, 1994.

20. T. Villa, T. Kam, R. K. Brayton, and A. L. Sangiovanni-Vincentelli. Explicit and Implicit Algorithms for Binate Covering Problems. IEEE Transactions on Computer Aided Design, vol. 16(7):677-691, July 1997.

21. S. Yang. Logic Synthesis and Optimization Benchmarks User Guide. Microelectronics Center of North Carolina, January 1991.

22. H. Zhang. SATO: An efficient propositional prover. In Proceedings of the International Conference on Automated Deduction, pages 272-275, July 1997.

\section{Authors' Vitae}

Vasco Manquinho

Obtained the BSc and MSc degrees at the Technical University of Lisbon, Portugal, in 1996 and 1999, respectively. He is currently working for the PhD degree at the Technical University of Lisbon. Since 2001, he has been an teaching assistant at the Computer Science Department of the Technical University of Lisbon, Portugal. His main research interests include Unate/Binate Covering, Integer Programming and Propositional Satisfiability. 
João Marques-Silva

Obtained the BSc and MSc degrees at the Technical University of Lisbon, Portugal, in 1988 and 1991, respectively, and the PhD degree at the University of Michigan, Ann Arbor, in 1995. Since 1995, he has been an Assistant Professor at the Computer Science Department of the Technical University of Lisbon, Portugal, and a member of the Cadence European Laboratories. His research interests include Algorithms for Discrete Optimization Problems, namely Satisfiability, Unate/Binate Covering and Integer Programming, and Applications of Discrete Optimization in EDA. 